\section{A 90-day Repeat-Dose Oral Toxicity Study of Yonkenafil Hydrochloride in Beagle Dogs}

\section{Abstract}

Yonkenafil hydrochloride, a novel synthetic phosphodiesterase type 5 inhibitor, is a promising drug for the treatment of erectile dysfunction. The repeated-dose toxicity of yonkenafil hydrochloride was assessed in male and female beagle dogs. Twenty-four dogs were randomly allocated to four groups and administered yonkenafil hydrochloride orally at dosages of $0,7,30$ or $120 \mathrm{mg} / \mathrm{kg} /$ day for 90 days followed by a 28-day recovery period. During the test period, clinical signs, mortality, body weight, food consumption, ophthalmoscopy, electrocardiography, hematology, serum biochemistry, urinalysis, organ weights, gross findings and histopathology were examined. There were no abnormal changes in the clinical observations except that gastrointestinal intolerance was observed in the 120 $\mathrm{mg} / \mathrm{kg} / \mathrm{day}$ group. The laboratory and histopathological examinations revealed yonkenafil hydrochloride toxicity to various organs, including the thyroid gland, liver, prostate, uterus and breast; at the end of the recovery period, this damage was resolved. The level of yonkenafil hydrochloride that resulted in no observable adverse effects in beagle dogs was $7 \mathrm{mg} / \mathrm{kg} /$ day.

Keywords: Yonkenafil hydrochloride; Erectile dysfunction; Toxicity; Clinical signs

Received: May 03, 2017; Accepted: May 16, 2017; Published: May 23, 2017

\section{Chang Liư ${ }^{1,2}$, Hongtao Jin ${ }^{1}$, Baodong Yu ${ }^{2}$, Jinfeng Wei ${ }^{1}$ and Aiping Wang ${ }^{1}$}

\author{
1 Institute of Materia Medica, Chinese \\ Academy of Medical Sciences \& Peking \\ Union Medical College, Beijing, PR \\ China \\ 2 Women's and Children's Hospital, \\ Qingdao University, Qingdao, PR China
}

\section{Corresponding author: Chang Liu}

\section{$\equiv$ Ich1001@yeah.net}

Institute of Materia Medica, Chinese Academy of Medical Sciences \& Peking Union Medical College, Beijing, PR China.

Tel: 86-18553263607

Citation: Liu C, Jin H, Yu B, et al. A 90day Repeat-Dose Oral Toxicity Study of Yonkenafil Hydrochloride in Beagle Dogs. J Biomedical Sci. 2017, 6:3.

\section{Introduction}

According to the definition of the National Institutes of Health Consensus Development Conference on Impotence, erectile dysfunction (ED), which will affect the lives of approximately 300 million men worldwide by the year 2025, is the inability to achieve or maintain an erection sufficient for satisfactory sexual performance [1,2]. Pharmacotherapy is currently the mainstay treatment for ED. According to the suggestion of the American Urological Association (AUA) guideline on ED, the first-line treatment of ED should be phosphodiesterase type 5 (PDE5) inhibitors, which include sildenafil, tadalafil and vardenafil [3]. Treatment of ED with PDE5 inhibitors is generally well tolerated, although some side effects, such as headache, flushing, dyspepsia, nasal congestion and unusual visual disturbances, have been reported $[4,5]$. PDE5 inhibitors are structurally similar to cGMP and compete with CGMP at the catalytic site of PDE5. Inhibition of PDE5 in human corpus cavernosum increases intracellular cGMP levels in trabecular smooth muscle cells to achieve relaxation and increased blood flow to the penis [6-9]. Recent studies have shown that PDE5 inhibitors have remarkable therapeutic effects on many other diseases, such as benign prostatic hyperplasia, pulmonary hypertension, and Alzheimer's disease [10-12].
With the widespread success and acceptance of these agents, major interest was generated in the potential of PDE5 inhibitors. Several new PDE5 inhibitors, such as avanafil, udenafil, SLx-2101 and mirodenafil, are candidates to enter the market in coming years [13]. Yonkenafil hydrochloride, a novel synthetic PDE5 inhibitor and analog of sildenafil, is a promising drug for the treatment of ED. Yonkenafil (IC50=2.0 nM) is pharmacologically more active than sildenafil $(I C 50=4.5 \mathrm{nM})$ and possesses fewer gastrointestinal side effects [14]. Previous research has also demonstrated that yonkenafil can efficiently reduce cerebral infarction, neurologic deficits, edema, and neuron damage in the infarct area $[14,15]$, and suppress the microglial activation to promote neuronal survival $[16,17]$. Yonkenafil has been submitted to China Food Drug Administration (CFDA) for the approval as a new drug, and is now undergoing clinical trials in China [18].

However, the information regarding the toxicity of yonkenafil is very limited. An understanding of the safety profile of yonkenafil is imperative to fully anticipate its therapeutic potential. This study was designed to evaluate the repeated-dose toxicity of yonkenafil hydrochloride in beagle dogs, with the purpose of 
obtaining information on the safety of yonkenafil hydrochloride. The study was performed according to "the Guidelines for Longterm Toxicity Tests of Chemicals" provided by the China Food and Drug Administration (CFDA) under Good Laboratory Practice Regulations. This study was conducted in accordance with the principles and procedures outlined in the National Institutes of Health Guide for the Care and Use of Laboratory Animals. This protocol was approved by The Animal Care \&Welfare Committee Institute of Materia Medica, CAMS\&PUMC.

\section{Materials and Methods}

\section{Test article}

Yonkenafil hydrochloride (Figure 1) (purity 99.40\%) is a watersoluble, odorless, yellow powder. The capsules $(300-500 \mathrm{mg}$, 200-300 mg, 40-50 mg yonkenafil hydrochloride/capsule) were obtained from Tianjin Tasly Company Ltd. (Tianjin, P.R. China) and were used for oral administration in this study. The number of capsules for each dog was calculated every week based on the recent body weight.

\section{Animals and husbandry}

Thirty-two healthy male and female Beagle dogs weighing 6-8 $\mathrm{kg}$ and aged 6-12 months were obtained from the Institute of Laboratory Animal Science, Peking Union Medical College \& Chinese Academy of Medical Sciences (Beijing, China). All dogs were acclimatized in the experimental facilities for a period of 4 weeks before the onset of the experiment. The animals were housed individually in cages and maintained in an environmentally controlled animal room. The animal room was monitored and maintained under a $12 \mathrm{~h}$ light-dark cycle controlled via an automatic timer with a temperature of $23 \pm 5^{\circ} \mathrm{C}$ and relative humidity between $40 \%$ and $60 \%$. The ventilation frequency was 15 times h-1 throughout this study. The dogs were provided ad libitum access to Certified Expanded Diet (Laboratory Animal Science Center, Academy of Military Medical Sciences, Beijing, China). Municipal water, processed through a reverse osmosis membrane, was provided to the dogs ad libitum.

\section{Study design and dose selection}

Male and female dogs ( $n=4 /$ sex/dose) were assigned randomly into four treatment groups $(0,7,30$ and $120 \mathrm{mg} / \mathrm{kg} /$ day yonkenafil hydrochloride). Two animals per sex per group were sacrificed under pentobarbital anesthesia at the end of the treatment period on Day 90, and the remaining two animals per sex per group were examined after a 28-day recovery period.

The selection of dosage levels in the current study was based on the results of an acute toxicity study of yonkenafil hydrochloride in mice and pharmacodynamic data from rats. In the acute study, the LD50 was $2000 \mathrm{mg} / \mathrm{kg} / \mathrm{day}$, and the 95\% confidence interval was from 1610 to $2438 \mathrm{mg} / \mathrm{kg} /$ day. In the pharmacodynamic study, the minimal effective dose was $6 \mathrm{mg} / \mathrm{kg} / \mathrm{day}$ and the maximal effective dose was $24 \mathrm{mg} / \mathrm{kg} /$ day. The optimal dose was $12 \mathrm{mg} / \mathrm{kg} /$ day. In the present study, $120 \mathrm{mg} / \mathrm{kg} /$ day was specified as the high-dose level, which was 33.3 times the optimal effective dosage. Lower doses of $30 \mathrm{mg} / \mathrm{kg} /$ day and $7 \mathrm{mg} / \mathrm{kg} /$ day were selected as mid- and low-dose levels, respectively.

\section{Clinical observation}

Mortality and clinical observations were recorded daily. The body weights and temperatures of all animals were measured twice during the quarantine period, and once every week during the administration and recovery periods. Food consumption was measured twice during the quarantine period and at weekly intervals during treatment and convalescence. The amounts of food (approximately $300 \mathrm{~g}$ ) were recorded before they were supplied to each cage, and the remaining food was measured the next day to determine the differences, which were regarded as daily food consumption (g per $100 \mathrm{~g}$ bodyweight).

Ophthalmoscopic examinations were performed on each dog before the start of treatment (Day -14, Day -7, i.e., 14 and 7 days before treatment initiation, respectively), during the treatment period (Day 45), after the last dose (Day 90) and after recovery (Day 118) using a binocular indirect ophthalmoscope (Topcon, Japan). The observation areas included the cornea, conjunctiva, sclera, iris, lens and fundus. Electrocardiograms were conducted on Day-14, Day-7, Day 45, Day 90 and Day 118 to measure the heart rate, P-R interval, QRS interval and QT interval.

\section{Laboratory testing parameters}

Blood samples were collected from all animals on Day-14, Day7, Day 45, Day 90 and Day 118. Urine samples were collected

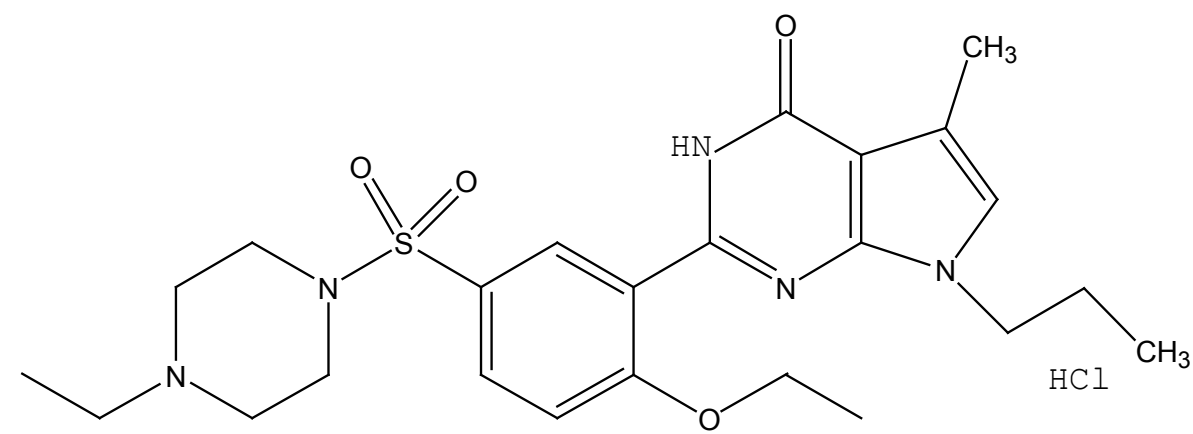

Figure 1 The structure of yonkenafil hydrochloride. Yonkenafil hydrochloride (2-(2-ethoxy-5-(4ethylpiperazin-1-yl sulfonyl) phenyl)-5-methyl-7-propyl-3H-pyrrolo[2, 3-d] pyrimidin -4(7H) -one- hydrochloride). Formula: $\mathrm{C} 24 \mathrm{H} 33 \mathrm{~N} 5 \mathrm{O} 4 \mathrm{~S} \cdot \mathrm{HCl}, \mathrm{MW}$ : 523.67. 
from all animals on Day 90 and Day 118. All dogs were fasted for more than $12 \mathrm{~h}$ prior to the blood/urine sample obtained. Blood samples were drawn from a forelimb vein into evacuated blood collection tubes. Under anesthesia, urine was collected from the bladder onto a specimen test paper through a catheter. EDTA and sodium citrate were used as anticoagulants for the blood coagulation study.

\section{Hematology and blood coagulation}

The hematological parameters were assessed using a MEK6318K Automated Hematology Analyzer (Nihon-Kodhen Co., Tokyo, Japan) for erythrocyte counts (RBC), hemoglobin ( $\mathrm{Hb})$, hematocrit (HCT), mean corpuscular volume (MCV), mean corpuscular hemoglobin ( $\mathrm{MCH})$, mean corpuscular hemoglobin concentration $(\mathrm{MCHC})$, red cell distribution width-variation coefficient (RDW-CV), platelet count (PL), mean platelet volume (MPV), platelet distribution width (PDW), plateletcrit (PCT), leukocyte counts (WBC) and differential cell count (DC). Blood smears were stained with Wright-Giemsa brilliant-cresyl-blue (Heath and Daland, 1931), and the reticulocyte count (Reti) was performed via light microscopy.

The coagulation parameters, including thrombin time (TT), prothrombin time (PT), activated partial thromboplastin time (APTT), and fibrinogen (Fbg), were evaluated with the DIAGNOSTICA STAGO STA-4 Coagmaster kit (Junior Instruments Co., Gennevilliers, France).

\section{Serum biochemistry}

The blood chemistry parameters were determined using a Vitalab Autoanalyzer (Vital Scientific, Dieren, the Netherlands) and an AVL-9181 Autoelectrolyte Analyzer (AVL Scientific Co., Roswell, Georgia, USA). The parameters measured were aspartate aminotransferase (AST), alanine aminotransferase (ALT), alkaline phosphatase (ALP), glucose (GLU), blood urea nitrogen (BUN), uric acid (UA), lactate dehydrogenase (LDH), total protein (TP), albumin (ALB), total bilirubin (T-BIL), total cholesterol (T-CHO), triglycerides (TG), creatinine (CRE), creatine kinase (CK), albuminglobulin ratio $(A / G)$, and serum levels of calcium (Ca), sodium $(\mathrm{Na})$, potassium $(\mathrm{K})$, and chloride $(\mathrm{Cl})$.

\section{Urinalysis}

Urinalysis was conducted using Multistrix ${ }^{\circledR}$ Strips (Bayer Corp., Bridgend, South Wales, UK) for parameters including glucose (GLU), bilirubin (BIL), ketones (KET), specific gravity (SG), occult blood (BLO), pH, protein (PRO), urobilinogen (URO), nitrites (NIT), leukocytes (LEU) and urinary sediment.

\section{Necropsy and histopathologic examination}

2 dogs/sex/group were sacrificed under ether anesthesia at the end of the treatment period, and the remaining two dogs/ sex/group were sacrificed at the end of the recovery period. A thorough necropsy was performed, and the following organs were excised and weighed: brain, lungs, heart, liver, spleen, thymus, kidneys, adrenal glands, prostate, testes, epididymitis, uterus and ovaries. The relative weight (weight per $100 \mathrm{~g}$ body weight) of each organ was calculated and recorded. The above organs and the thyroid gland, parathyroid glands, salivary glands, gall bladder, pancreas, sternum and bone marrow, spinal cord, pituitary gland, esophagus, stomach, intestines (duodenum, jejunum, ileum and colon), lymph nodes (mesenteric), urinary bladder, breast, trachea, sciatic nerve and optic nerve were fixed in $10 \%$ buffered formalin solution. These samples were routinely processed, embedded in paraffin, sectioned at $5 \mu \mathrm{m}$, and stained with hematoxylin-eosin for histopathological examination.

\section{Toxicokinetics}

A concomitant toxicokinetics (TK) study was performed in the yonkenafil hydrochloride-treated groups. An Agilent 1100 series HPLC system (Agilent Technologies, Palo Alto, CA, USA) coupled to an Applied Biosystems Sciex Q-trap ${ }^{\text {TM }}$ mass spectrometer (Concord, Ontario, Canada) via an electrospray ionization (ESI) source was used for analysis. Applied Biosystems Analyst software package version 1.3.2 was used to control the LC-MS/ MS system and for data acquisition and processing. Yonkenafil hydrochloride and the I.S. (internal standard) (diazepam $250 \mathrm{ng} /$ $\mathrm{mL}$ ) were separated on a $5 \mu \mathrm{m}$ Zorbax Extend $\mathrm{C}_{18}$ column (150 $\times 4.6 \mathrm{~mm}$ I.D. from Agilent Technologies) maintained at $30^{\circ} \mathrm{C}$. The mobile phase consisted of $85 \%$ methanol and $15 \% 10 \mathrm{mM}$ ammonium acetate buffer delivered at a flow rate of $1.0 \mathrm{~mL} / \mathrm{min}$. A two-phase switching valve was used to divert the pre-eluent from entering the ion source. An approximately 1:1 split of the column eluent was included so that only $0.5 \mathrm{~mL} / \mathrm{min}$ entered the mass spectrometer. The mass spectrometer was operated in the positive ESI mode with multiple reaction monitoring (MRM) at unit resolution. Nitrogen was used as the nebulizer, heater, curtain gas and collision activation dissociation (CAD) gas. The precursorto-product ion transitions were monitored at $\mathrm{m} / \mathrm{z} 488.4 \rightarrow 99.2$ and $m / z \quad 488.1 \rightarrow 310.1$ for yonkenafil hydrochloride and at $\mathrm{m} / \mathrm{z} 285.2 \rightarrow \mathrm{m} / \mathrm{z} 193.2$ for diazepam. The mass spectrometer instrumental parameters were tuned to maximize the generation of precursor and fragment ions by infusion of a solution of yonkenafil hydrochloride and I.S. into the ESI source at $10 \mu \mathrm{L} / \mathrm{min}$. The optimum parameters were as follows: nebulizer $\left(\mathrm{GS}_{1}\right)$, heater $\left(\mathrm{GS}_{2}\right)$ and curtain gas flow rates: 55, 40 and 15 units, respectively; ion spray needle voltage: $1500 \mathrm{~V}$; heater gas temperature: $450^{\circ} \mathrm{C}$; collision gas: $\left(\mathrm{N}_{2}\right)$ medium; declustering potential: $85 \mathrm{~V}$; collision energies: $83 \mathrm{eV}(\mathrm{m} / z 488.4 \rightarrow 99.2)$ and $39 \mathrm{eV}(\mathrm{m} / z$ 488.4 $\rightarrow$ 310.3) for yonkenafil and $44 \mathrm{eV}$ for diazepam.

Blood samples were obtained on the $1^{\text {st }}$ day $(0.5,1,2,3,4,5$, $6,7,8,24 \mathrm{~h}$ after dosing), $45^{\text {th }}$ day $(0.5,5,24 \mathrm{~h}$ after dosing), $90^{\text {th }}$ day $\left(0.5,5,24,48,72,96 \mathrm{~h}\right.$ after dosing) and $118^{\text {th }}$ day for plasma yonkenafil hydrochloride level determinations. On each occasion, approximately $2.0 \mathrm{~mL}$ of blood was drawn from the foreleg vein of the dogs; the samples were collected in tubes containing heparin. The blood samples were centrifuged immediately, and the plasma samples were stored in a $-20^{\circ} \mathrm{C}$ freezer until analysis. After thawing at room temperature, the plasma samples were vortexed and an aliquot $(100 \mu \mathrm{L})$ was mixed with $100 \mu \mathrm{L}$ I.S. (internal standard) (diazepam $250 \mathrm{ng} / \mathrm{mL}$ ) and $100 \mu \mathrm{L}$ methanol:water (50:50, v/v) and $50 \mu \mathrm{L} \mathrm{NaOH}(1 \mathrm{M})$ in a $10 \mathrm{~mL}$ capped tube. The mixture was vortexed for $10 \mathrm{~s}$ and was extracted with $3.0 \mathrm{~mL}$ diethylether:dichloromethane, 60:40, v/v). 
After shaking for $10 \mathrm{~min}$ and centrifuging at $3500 \times \mathrm{g}$ for $5 \mathrm{~min}$, the upper organic phase was carefully transferred to another tube and evaporated at $40^{\circ} \mathrm{C}$ under a gentle stream of nitrogen. The residue was reconstituted in $200 \mu \mathrm{L}$ mobile phase, and 20 $\mu \mathrm{L}$ was injected into the LC-MS/MS system. TK determination was conducted at the College of Life Science of Jilin University (Changchun, China).

\section{Statistical analysis}

The experimental groups were compared against the control group in terms of body weight, food consumption, hematological parameters (except for the differential leukocyte count and reticulocyte count), blood chemistry values, and organ weights. If the data were homogenous according to Bartlett's test, a oneway analysis of variance (ANOVA) was used. If the data were not homogeneous (and for the differential leukocyte count and reticulocyte count), Kruskal-Wallis's test was employed. The parameters found to be significant in the one-way analysis of variance (ANOVA) were assessed by the Dunnett test. Percentage and ratio data from the urinalysis were evaluated by chi-square tests. Significance levels for pair-wise comparisons were reported at $p<0.05$ or $p<0.01$.

\section{Results}

\section{Clinical observations}

All beagle dogs survived until the scheduled necropsy. In 120 $\mathrm{mg} / \mathrm{kg} /$ day group, emesis occurred 2-5 h after yonkenafil hydrochloride administration in three dogs (one male and two females) on Day 1 . One male dog in the $120 \mathrm{mg} / \mathrm{kg} /$ day group became emaciated after Day 17, and penile erection was observed frequently. Polysialia and soft feces were observed in three dogs in the high-dose group after Day 20, and these symptoms disappeared a few days later. No other abnormal clinical signs were observed in other groups (Table 1).

Group mean body weight was statistically significantly decreased after 6 and 13 weeks of dosing in the male dogs in the high-dose group compared with the control group (decreases of $24 \%$ and $34 \%$, respectively). However, these changes were not observed in female dogs. The weight changes are presented in Figures $\mathbf{2}$ and 3. The mean daily food consumption was comparable between the control and treatment groups in both genders. There was no significant change in body temperature, and the numerical values of their temperatures were in the normal range. Increases in eye discharge were observed in the $120 \mathrm{mg} / \mathrm{kg} /$ day group during the treatment period; however, these symptoms resolved by the end of recovery. The electrocardiographic examination (heart rate, P-R interval, QRS interval, QT interval) did not indicate any treatment-related adverse effects

\section{Laboratory Investigations}

\section{Hematology and coagulation}

On Days 45 and 90, there were no statistically significant differences in any of the hematology parameters between any of the treatment groups and the control group for either sex. Fibrinogen levels were statistically significantly increased in the male dogs at the 7 and $30 \mathrm{mg} / \mathrm{kg} /$ day doses on Day 90 compared to the control group (Table 2). The significance was, however, due to the low value in the control group and, therefore, was not considered treatment-related. In addition, the Fbg values at the 7 and $30 \mathrm{mg} / \mathrm{kg} /$ day doses were comparable to pretest and Day 45 values.

\section{Serum biochemistry}

Blood chemistry tests revealed no statistical differences between the groups before treatment initiation. On Day 45, the GLU value of male beagles slightly increased at 7 and $30 \mathrm{mg} / \mathrm{kg} /$ day groups ( $4.76 \pm 0.73$ and $4.63 \pm 0.13$, respectively) compared with the value at $0 \mathrm{mg} / \mathrm{kg} /$ day group $(3.31 \pm 0.11)$. Increased GLU values at 7 and $30 \mathrm{mg} / \mathrm{kg} /$ day groups were also found on Day 90(4.79 $\pm 0.41,4.10 \pm 0.11$ and $3.26 \pm 0.08$, for low-, mid- and control group, respectively). However, these minor changes were within the normal ranges. Decreased $A L B$ and $A / G$ and increased $C K$, AST and LDH at $120 \mathrm{mg} / \mathrm{kg} /$ day male beagles were observed on Day 90 , and these parameters returned to normal at the end of the recovery period (Tables $\mathbf{2}$ and $\mathbf{3}$ ). No other abnormal findings were observed.

\section{Urinalysis}

On Day 90 and Day 118, the indexes of PRO, BIL and KET were positive in all yonkenafil hydrochloride groups (data not shown). The changes observed in the urinalysis could not be definitively declared as indicating yonkenafil hydrochloride-related toxicity.

\section{Organ weights}

The selected absolute organ weight and organ-to-terminal body weight ratio (relative organ weight) are summarized in Table 4. At the end of dosing, the absolute and relative uterus weights were increased in females at 7, 30 and $120 \mathrm{mg} / \mathrm{kg} /$ day compared with the control group. The absolute and relative liver weights were increased in males at the high dose. Decreased absolute prostate weight was observed in the high-dose group. At the end of recovery, there were no notable differences in absolute and relative organ weights in either males or females.

Table 1 Effect of yonkenafil hydrochloride on clinical symptoms in beagle dogs during the 90-day study.

\begin{tabular}{|c|c|c|c|c|c|c|c|c|c|c|}
\hline Findings & \multicolumn{3}{|c|}{ Males } & \multicolumn{1}{c|}{ Females } \\
\hline & Control & Low & Medium & High & Control & Low & Medium & High \\
\hline & $(n=4)$ & $(n=4)$ & $(n=4)$ & $(n=4)$ & $(n=4)$ & $(n=4)$ & $(n=4)$ & $(n=4)$ \\
\hline Emesis & 0 & 0 & 0 & 1 & 0 & 0 & 0 & 2 \\
\hline Emaciation & 0 & 0 & 0 & 1 & 0 & 0 & 0 & 0 \\
\hline Polysialia and soft feces & 0 & 0 & 0 & 1 & 0 & 0 & 0 & 2 \\
\hline
\end{tabular}




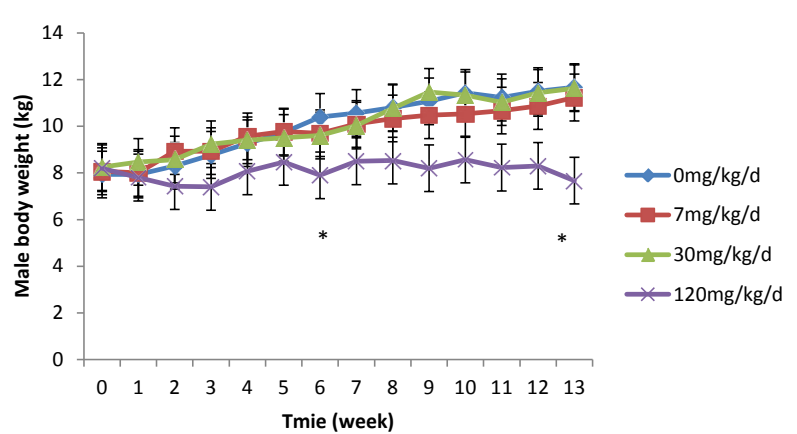

Figure 2 Effect of yonkenafil hydrochloride on the body weight of male dogs during the 90-day toxicity study. All data are mean \pm SD (3 dogs/group/sex). ${ }^{*} p<0.05$.

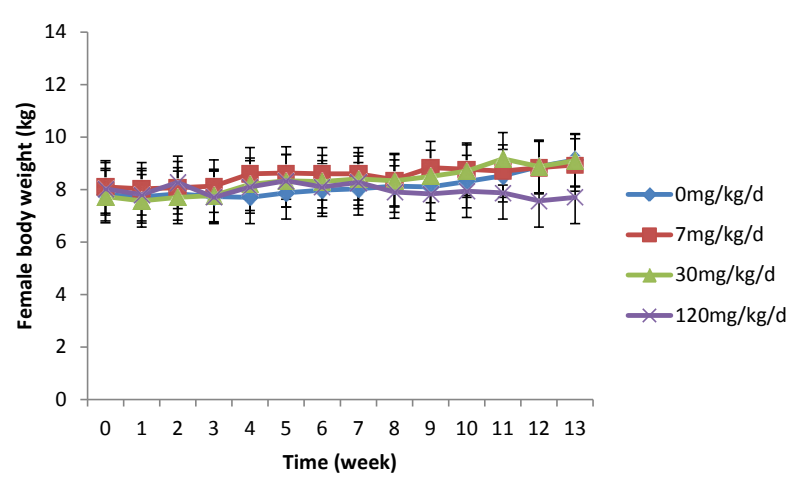

Figure 3 Effect of yonkenafil hydrochloride on the body weight of female dogs during the 90-day toxicity study. All data are mean \pm SD (3 dogs/group/sex).

\section{Pathology}

\section{Gross examination}

During necropsy after the last dose, hematomas were found at the edge of the spleen in dogs in the control and low-dose groups. In one female dog, one ovary was atrophied in the $120 \mathrm{mg} / \mathrm{kg} /$ day group. In the $120 \mathrm{mg} / \mathrm{kg} /$ day group, a goiter was found in two male dogs. At the end of recovery, the dogs examined showed no abnormalities (Table 5).

\section{Histopathological examination}

After the treatment period, hematomas at the edge of the spleen and parafollicular hyperplasia of the thyroid gland were found in the control group. Parafollicular hyperplasia was also seen in the 7 and $30 \mathrm{mg} / \mathrm{kg} /$ day groups. In the $120 \mathrm{mg} / \mathrm{kg} /$ day yonkenafil hydrochloride group, hepatic steatosis, parafollicular hyperplasia, endometrial hyperplasia, hyperplasia of mammary glands and acinar epithelial degeneration of the prostate were observed (Table 6). At the end of recovery, a hematoma was found at the edge of the spleen in one dog in the $0 \mathrm{mg} / \mathrm{kg} / \mathrm{day}$ group. In the $120 \mathrm{mg} / \mathrm{kg} /$ day group, degenerative changes in the prostate and hyperplasia of the mammary glands were also observed, but the pathological lesions were partially alleviated. No abnormal changes were found in other groups (Table 7).

\section{Toxicokinetics}

Systemic exposure in beagle dogs was indicated by the plasma $A \cup C_{0-t}$ and $C_{\max }$. There were significant differences in the pharmacokinetic parameters of yonkenafil hydrochloride between males and females during the 90 -day oral administration for the mid-and high-dose groups. The $A U C_{0-t^{\prime}} A U C_{0-\infty}$ and $C_{\max }$ were much lower in females than in males. The plasma concentration of yonkenafil hydrochloride on the Day 1, Day 45 and Day 90 demonstrated a dose-dependent and linear increase $(r>0.798$, $\mathrm{p}<0.01$ ) with maximum plasma values observed at approximately $2 \mathrm{hr}$ after administration. No significant accumulation of exposure level was observed after 45 or 90 days of administration. No plasma concentration of yonkenafil hydrochloride was detected after the 28-day recovery period.

\section{Discussion}

Since sildenafil's approval by the US Food and Drug Administration (FDA) in March 1998, the concept of PDE5 inhibitors for the treatment of ED has gained tremendous interest [19]. Yonkenafil hydrochloride, a novel synthetic PDE5 inhibitor that is structurally similar to CGMP, has been evaluated for its potential clinical use for ED. As part of a non-clinical safety program to evaluate yonkenafil hydrochloride, the current study was conducted to assess the potential repeated-dose toxicity of yonkenafil hydrochloride in beagle dogs.

In this 90-day oral toxicity study conducted with male and female beagles, all treated and control animals survived the experimental period in good general health. The mean body weights and body weight gain of the treated male dogs in the $120 \mathrm{mg} / \mathrm{kg} /$ day group significantly decreased compared with the control group. By contrast, the body weight and body weight gain of females were comparable to the controls throughout the experiment. No significant changes in food intake were observed in male or female dogs or among treatment groups. In the absence of other observations associated with systemic toxicity and lower feed intake, the magnitude of the body weight decrease in the highdose group males compared with the controls exceeded $34 \%$ at Day 90 . This finding might indicate that the dogs challenged with the high drug dose reached or exceeded the maximum tolerated dose.

At the high dose $(120 \mathrm{mg} / \mathrm{kg} /$ day $)$, emesis, emaciation, transient salivation and soft feces were observed in a few dogs, and these symptoms disappeared a few days later. These results indicate that yonkenafil hydrochloride induced gastrointestinal intolerance at the high dose. We did not observe gastrointestinal pathological changes in the histopathological examination. Thus, the effect of yonkenafil hydrochloride in the gastrointestinal tract is at the functional level. Similarly, gastrointestinal intolerance was observed in dogs that received sildenafil in 1-month and 6-month preclinical studies [20].

Significant differences were observed in the results of the biochemical parameters of male dogs in the $120 \mathrm{mg} / \mathrm{kg} /$ day dose group. Lower ALB and A/G and higher AST, LDH were observed on Day 90 in males in the high-dose group compared with the control group. Moreover, increased absolute and relative liver 
Table 2 Selected laboratory parameters of male dogs after 90-day administration of yonkenafil hydrochloride and 28-day recovery.

\begin{tabular}{|c|c|c|c|c|c|c|c|}
\hline & Dose & Male & & & & & \\
\hline & (mg/kg/day) & $\operatorname{ALB}(\mathrm{g} / \mathrm{L})$ & CK (U/L) & $\mathrm{LDH}(\mathrm{U} / \mathrm{L})$ & $A / G$ & AST (U/L) & $\mathrm{Fbg}(\mathrm{mg} / \mathrm{dl})$ \\
\hline \multirow[t]{4}{*}{$\mathrm{D}-7 \mathrm{a}$} & 0 & $32.3 \pm 0.9$ & $352.0 \pm 75.4$ & $153.7 \pm 66.5$ & $1.04 \pm 0.11$ & $36.3 \pm 5.1$ & $272.3 \pm 32.5$ \\
\hline & 7 & $32.1 \pm 1.9$ & $396.7 \pm 63.9$ & $112.3 \pm 16.6$ & $1.09 \pm 0.06$ & $40.0 \pm 8.5$ & $262.0 \pm 87.1$ \\
\hline & 30 & $32.6 \pm 1.1$ & $445.7 \pm 78.7$ & $195.7 \pm 61.7$ & $1.08 \pm 0.05$ & $42.7 \pm 4.9$ & $268.5 \pm 18.6$ \\
\hline & 120 & $32.2 \pm 0.3$ & $467.7 \pm 59.2$ & $166.7 \pm 63.7$ & $1.09 \pm 0.15$ & $41.0 \pm 1.0$ & $316.3 \pm 39.7$ \\
\hline \multirow[t]{4}{*}{ D 45a } & 0 & $34.8 \pm 0.7$ & $327.0 \pm 82.1$ & $149.7 \pm 21.2$ & $1.18 \pm 0.08$ & $34.3 \pm 1.52$ & $233.3 \pm 23.9$ \\
\hline & 7 & $32.8 \pm 0.8$ & $331.7 \pm 68.4$ & $111.0 \pm 31.1$ & $1.12 \pm 0.02$ & $40.3 \pm 12.7$ & $251.3 \pm 30.8$ \\
\hline & 30 & $33.4 \pm 1.0$ & $369.7 \pm 40.3$ & $175.3 \pm 27.5$ & $1.13 \pm 0.08$ & $39.7 \pm 1.2^{*}$ & $266.6 \pm 31.6$ \\
\hline & 120 & $30.5 \pm 2.6$ & $458.0 \pm 94.4$ & $286.3 \pm 128.4$ & $0.92 \pm 0.17$ & $41.3 \pm 7.2$ & $238.9 \pm 28.8$ \\
\hline \multirow[t]{4}{*}{ D 90a } & 0 & $34.7 \pm 1.7$ & $244.3 \pm 36.9$ & $114.0 \pm 11.3$ & $1.19 \pm 0.04$ & $35.0 \pm 2.0$ & $165.4 \pm 20.7$ \\
\hline & 7 & $33.30 \pm 1.11$ & $233.0 \pm 50.9$ & $77.7 \pm 25.4$ & $1.27 \pm 0.04$ & $41.0 \pm 7.0$ & $260.1 \pm 48.3^{*}$ \\
\hline & 30 & $33.2 \pm 1.2$ & $298.7 \pm 59.0$ & $131.3 \pm 36.1$ & $1.06 \pm 0.08$ & $45.7 \pm 5.7^{*}$ & $260.4 \pm 16.0^{*}$ \\
\hline & 120 & $27.5 \pm 3.6^{*}$ & $561.3 \pm 41.9 * *$ & $386.0 \pm 53.4^{* *}$ & $0.88 \pm 0.14 *$ & $82.7 \pm 27.2^{* *}$ & $324.6 \pm 59.1 *$ \\
\hline \multirow[t]{4}{*}{ D 118b } & 0 & 32.1 & 189 & 63 & 1.05 & 28 & 236.1 \\
\hline & 7 & 32.3 & 267 & 77 & 0.99 & 50 & 211.6 \\
\hline & 30 & 31.3 & 205 & 67 & 0.92 & 44 & 208.2 \\
\hline & 120 & 27.9 & 222 & 97 & 0.9 & 33 & 278.8 \\
\hline
\end{tabular}

Table 3 Selected laboratory parameters of female dogs after 90-day administration of yonkenafil hydrochloride and 28-day recovery.

\begin{tabular}{|c|c|c|c|c|c|c|c|}
\hline & Dose & Female & & & & & \\
\hline & (mg/kg/day) & ALB (g/L) & CK (U/L) & LDH (U/L) & $A / G$ & AST (U/L) & $\mathrm{Fbg}(\mathrm{mg} / \mathrm{dl})$ \\
\hline \multirow[t]{4}{*}{$D-7 a$} & 0 & $33.4 \pm 1.4$ & $377.7 \pm 125.9$ & $158.3 \pm 48.2$ & $1.03 \pm 0.13$ & $41.3 \pm 2.1$ & $275.4 \pm 66.1$ \\
\hline & 7 & $35.4 \pm 1.9$ & $279.3 \pm 83.1$ & $101.3 \pm 30.5$ & $1.18 \pm 0.09$ & $40.7 \pm 8.0$ & $248.9 \pm 27.5$ \\
\hline & 30 & $33.9 \pm 1.3$ & $399.0 \pm 125.0$ & $123.3 \pm 42.7$ & $1.13 \pm 0.14$ & $40.3 \pm 8.5$ & $249.8 \pm 19.0$ \\
\hline & 120 & $34.6 \pm 1.8$ & $296.0 \pm 86.6$ & $119.3 \pm 25.7$ & $1.19 \pm 0.04$ & $40.7 \pm 6.0$ & $250.3 \pm 30.9$ \\
\hline \multirow[t]{4}{*}{ D 45 a } & 0 & $32.6 \pm 3.8$ & $369.7 \pm 130.0$ & $211.0 \pm 102.6$ & $1.06 \pm 0.26$ & $39.0 \pm 9.5$ & $231.6 \pm 24.1$ \\
\hline & 7 & $34.5 \pm 0.6$ & $231.0 \pm 79.8$ & $100.3 \pm 29.7$ & $1.17 \pm 0.03$ & $41.0 \pm 11.8$ & $244.4 \pm 48.9$ \\
\hline & 30 & $33.4 \pm 0.7$ & $333.0 \pm 66.57$ & $152.3 \pm 41.5$ & $1.09 \pm 0.11$ & $36.3 \pm 4.0$ & $268.5 \pm 62.8$ \\
\hline & 120 & $32.9 \pm 1.0$ & $271.0 \pm 123.0$ & $133.0 \pm 49.3$ & $1.05 \pm 0.14$ & $34.00 \pm 11.3$ & $273.2 \pm 59.1$ \\
\hline \multirow[t]{4}{*}{ D 90 a } & 0 & $34.2 \pm 1.00$ & $260.0 \pm 103.1$ & $102.3 \pm 19.1$ & $1.26 \pm 0.09$ & $36.7 \pm 10.2$ & $238.6 \pm 32.7$ \\
\hline & 7 & $34.3 \pm 1.7$ & $182.67 \pm 17.6$ & $70.7 \pm 22.3$ & $1.87 \pm 0.19$ & $42.0 \pm 7.9$ & $272.8 \pm 56.8$ \\
\hline & 30 & $33.2 \pm 2.1$ & $225.0 \pm 21.0$ & $97.3 \pm 20.6$ & $1.09 \pm 0.13$ & $34.7 \pm 5.5$ & $244.7 \pm 39.1$ \\
\hline & 120 & $30.7 \pm 2.7$ & $331.7 \pm 128.1$ & $130.0 \pm 41.7$ & $1.00 \pm 0.19$ & $38.7 \pm 12.1$ & $305.8 \pm 85.9$ \\
\hline \multirow[t]{4}{*}{ D $118 b$} & 0 & 30.8 & 155 & 52 & 1.01 & 22 & 186.9 \\
\hline & 7 & 33.4 & 166 & 37 & 1.19 & 36 & 228.6 \\
\hline & 30 & 33.3 & 183 & 79 & 1.08 & 34 & 217.2 \\
\hline & 120 & 30.6 & 126 & 54 & 1.03 & 26 & 195.8 \\
\hline
\end{tabular}

a) Values are presented as means $\pm S D$. The number of animals in each group was $4 /$ sex for 90 -day treatment period.

b) The number of animals in each group was $2 /$ sex for 28 -day recovery period.

weights and the finding of hepatic steatosis were observed in the high dose yonkenafil hydrochloride-treated males. These changes may indicate that hepatic toxicity is caused by yonkenafil hydrochloride. After the 28-day recovery period, all of these changes returned to normal, demonstrating that the hepatic toxicity caused by a high dose of yonkenafil hydrochloride is reversible.

According to the coagulation test, an increase in fibrinogen was found in all yonkenafil hydrochloride-treated groups on Day 90, and the parameter returned to normal after the recovery period. Because this change was within the normal historical range of the laboratory, we did not consider this parameter to be related to yonkenafil hydrochloride toxicity.
Organ weight changes have long been considered a sensitive marker of chemically induced organ damage [21]. In the present study, elevated absolute and relative uterus weights were observed in all yonkenafil-treated groups. In the histopathological examination, endometrial hyperplasia of the uterus was observed in the high-dose group. Although the changes resolved after the recovery period, these findings suggest that this drug might affect the uterus after long-term treatment. Meanwhile, decreased absolute prostate weights were found in the highdose group. Combined with the finding of degenerative change in the prostate in histopathological examination, we concluded that the prostate was also a target organ for toxicity. 
Table 4 Selected absolute organ weights (AW) and relative organ weights (RW) of dogs after 90 days of administration of yonkenafil hydrochloride and 28-day recovery.

\begin{tabular}{|c|c|c|c|c|c|c|c|}
\hline & Organ & Time & Detection item & $0 \mathrm{mg} / \mathrm{kg} /$ day & $7 \mathrm{mg} / \mathrm{kg} /$ day & $30 \mathrm{mg} / \mathrm{kg} / \mathrm{day}$ & $120 \mathrm{mg} / \mathrm{kg} / \mathrm{day}$ \\
\hline \multirow{8}{*}{ Male } & \multirow{4}{*}{ Liver } & D90 a & AW & 248.23 & 269.85 & 244.31 & 277.55 \\
\hline & & $\mathrm{D} 118 \mathrm{~b}$ & & 285.31 & 235.39 & 264.66 & 245.04 \\
\hline & & $\mathrm{D} 90 \mathrm{a}$ & RW & 2 & 2.4 & 2.2 & 3.5 \\
\hline & & D118 b & & 2.6 & 2 & 2 & 2.6 \\
\hline & Prostate & D118 b & AW & 6.28 & 7.73 & 3.74 & 2.61 \\
\hline & & D 90 a & & 4.25 & 4.91 & 5.3 & 4.24 \\
\hline & & D118 b & RW & 0.03 & 0.03 & 0.03 & 0.03 \\
\hline & & D118 b & & 0.04 & 0.04 & 0.04 & 0.05 \\
\hline \multirow[t]{8}{*}{ Female } & Liver & $\mathrm{D} 90 \mathrm{a}$ & AW & 215.32 & 223.58 & 212.39 & 224.75 \\
\hline & & D118 b & & 208.47 & 177.83 & 191.96 & 201.33 \\
\hline & & D 90 a & RW & 2.4 & 2.3 & 2.1 & 2.7 \\
\hline & & $\mathrm{D} 118 \mathrm{~b}$ & & 2.6 & 2.3 & 2.8 & 2.5 \\
\hline & Uterus & $\mathrm{D} 90 \mathrm{a}$ & AW & 2.03 & 14 & 11.16 & 8.26 \\
\hline & & $\mathrm{D} 118 \mathrm{~b}$ & & 5.66 & 3.2 & 1.38 & 3.33 \\
\hline & & D 90 a & RW & 0.02 & 0.25 & 0.12 & 0.12 \\
\hline & & D118 b & & 0.06 & 0.04 & 0.02 & 0.04 \\
\hline
\end{tabular}

a) Values are presented as means. The number of animals in each group was $2 /$ sex for 90 -day treatment period.

b) The number of animals in each group was $2 /$ sex for 28 -day recovery period

Table 5 Summary of gross findings after a 90-day oral administration of 7, 30 or $120 \mathrm{mg} / \mathrm{kg} /$ day to beagle dogs.

\begin{tabular}{|c|c|c|c|c|c|c|c|c|c|}
\hline \multirow[t]{3}{*}{ Organ } & \multirow[t]{3}{*}{ Findings } & \multicolumn{4}{|c|}{ Males } & \multicolumn{4}{|c|}{ Females } \\
\hline & & Control & Low & Medium & High & Control & Low & Medium & High \\
\hline & & $(n=2)$ & $(n=2)$ & $(n=2)$ & $(n=2)$ & $(n=2)$ & $(n=2)$ & $(n=2)$ & $(n=2)$ \\
\hline Thyroid gland & Goiter & 0 & 0 & 0 & 2 & 0 & 0 & 0 & 0 \\
\hline Spleen & Hematoma & 1 & 1 & 0 & 0 & 0 & 0 & 0 & 0 \\
\hline Ovary & Atrophy & - & - & - & - & 0 & 0 & 0 & 1 \\
\hline
\end{tabular}

Table 6 Summary of histopathological findings after a 90-day oral administration of 7, 30 or $120 \mathrm{mg} / \mathrm{kg} /$ day to beagle dogs.

\begin{tabular}{|c|c|c|c|c|c|c|c|c|c|}
\hline \multirow[t]{3}{*}{ Organ } & \multirow[t]{3}{*}{ Findings } & \multicolumn{4}{|c|}{ Males } & \multicolumn{4}{|c|}{ Females } \\
\hline & & Control & Low & Medium & High & Control & Low & Medium & High \\
\hline & & $(n=2)$ & $(n=2)$ & $(n=2)$ & $(n=2)$ & $(n=2)$ & $(n=2)$ & $(n=2)$ & $(n=2)$ \\
\hline Spleen & Hematoma & 1 & 0 & 0 & 0 & 0 & 0 & 0 & 0 \\
\hline Thyroid gland & Parafollicular hyperplasia & 1 & 1 & 1 & 2 & 0 & 0 & 0 & 0 \\
\hline Liver & Hepatic steatosis & 0 & 0 & 0 & 1 & 0 & 0 & 0 & 0 \\
\hline Uterus & Endometrial hyperplasia & - & - & - & - & 0 & 0 & 0 & 2 \\
\hline $\begin{array}{l}\text { Mammary } \\
\text { gland }\end{array}$ & Hyperplasia & - & - & - & - & 0 & 0 & 0 & 2 \\
\hline Prostate & Epithelial degenerative changes & 0 & 0 & 0 & 2 & - & - & - & - \\
\hline
\end{tabular}

In the gross examination, hematomas were found at the edge of the spleen in dogs in the 0 and $7 \mathrm{mg} / \mathrm{kg} /$ day groups. Additionally, in the histopathological examination on Day 90, the change was only found in one dog in control group, and another one dog in control group was also observed hematoma in spleen after 28day recovery period. We considered this change was due to the artifact of the necropsy procedure.

At the end of the treatment period, hepatic steatosis, parafollicular hyperplasia, endometrial hyperplasia of the uterus, hyperplasia of the mammary glands and degenerative changes in the prostate were observed in the high-dose group. Because parafollicular hyperplasia was also found in the control group, further clarification is required to determine whether the thyroid changes were induced by yonkenafil hydrochloride. At the end of recovery, no abnormal histopathological findings were observed in the liver, thyroid gland or uterus. Although degenerative changes in the prostate and hyperplasia of the mammary glands were found in the high-dose group, the degree and range of the pathological lesions were alleviated, indicating the reversibility of toxicity.

From the results in this study and in previous reports, we conclude that there are both differences and similarities between the toxicity of yonkenafil hydrochloride and other PDE5 inhibitors. Species-specific effects of sildenafil in dogs (beagle pain syndrome, male dogs), mice (marked intestinal dilatation) and rats (adaptive reversible hepatocellular hypertrophy associated 
Table 7 Summary of histopathological findings after a 28-day recovery period.

\begin{tabular}{|c|c|c|c|c|c|c|c|c|c|}
\hline \multirow[t]{3}{*}{ Organ } & \multirow[t]{3}{*}{ Findings } & \multicolumn{4}{|c|}{ Males } & \multicolumn{4}{|c|}{ Females } \\
\hline & & Control & Low & Medium & High & Control & Low & Medium & High \\
\hline & & $(n=2)$ & $(n=2)$ & $(n=2)$ & $(n=2)$ & $(n=2)$ & $(n=2)$ & $(n=2)$ & $(n=2)$ \\
\hline Spleen & Hematoma & 0 & 0 & 0 & 0 & 1 & 0 & 0 & 0 \\
\hline $\begin{array}{l}\text { Thyroid } \\
\text { gland }\end{array}$ & Parafollicular hyperplasia & 0 & 0 & 0 & 0 & 0 & 0 & 0 & 0 \\
\hline Liver & Hepatic steatosis & 0 & 0 & 0 & 0 & 0 & 0 & 0 & 0 \\
\hline Uterus & Endometrial hyperplasia & - & - & - & - & 0 & 0 & 0 & 0 \\
\hline $\begin{array}{l}\text { Mammary } \\
\text { gland }\end{array}$ & Hyperplasia & - & - & - & - & 0 & 0 & 0 & 1 \\
\hline Prostate & Epithelial degenerative changes & 0 & 0 & 0 & 1 & - & - & - & - \\
\hline
\end{tabular}

with secondary thyroid hypertrophy) were found in a few longterm studies [20]. The most common adverse effects of sildenafil and yonkenafil in beagle dogs were $\mathrm{GI}$ intolerance, such as emesis and transient salivation. Hepatic steatosis and parafollicular hyperplasia of the thyroid were also observed in yonkenafiltreated male dogs. Further study is needed to explain whether there is a relationship between hepatic steatosis and thyroid hypertrophy induced by yonkenafil hydrochloride. Additionally, yonkenafil hydrochloride may affect reproductive organs such as the prostate, uterus and breast. However, these changes were resolved or attenuated after a 28-day recovery period. The reasons for the differences between yonkenafil hydrochloride and other PDE5 inhibitors remain unknown, and further research concerning the mechanisms is needed.

Under the conditions of our study, the animals in the $120 \mathrm{mg} /$ $\mathrm{kg} /$ day yonkenafil hydrochloride group demonstrated toxic effects on the target organs, including the thyroid gland, liver, prostate, uterus and breast. It is notable that these effects (except damage to the uterus and breast) were only observed in male dogs. According to the toxicokinetic results, these differences may due to gender differences in drug metabolism. Other PDE5 inhibitors, such as sildenafil, vardenafil and avanafil, undergo hepatic metabolism via the CYP3A4 pathway, but no gender difference was reported [21-23]. Thus, further research is needed concerning the mechanism of action and metabolism of yonkenafil hydrochloride. Most of the changes caused by yonkenafil hydrochloride were resolved or attenuated by the end of the recovery period, suggesting that the damage to these organs is reversible.

Based on the adverse effects of the clinical findings, laboratory examination and the pathology observed in the $120 \mathrm{mg} / \mathrm{kg} /$ day treatment group, the NOAEL of yonkenafil hydrochloride was considered to be $7 \mathrm{mg} / \mathrm{kg} /$ day under the present experimental conditions in beagle dogs.

\section{Acknowledgements}

We are grateful for the generous gift of yonkenafil hydrochloride provided by Tianjin Tasly Company Ltd. (Tianjin, P.R. China). The authors also wish to thank Professor Xueyong $\mathrm{Li}$ and $\mathrm{Ms} \mathrm{Hui} \mathrm{Li}$, Jin Li for their technical aid on experimental animals and Ms Yan Liu and Mr Chenghe Li for technical assistance on histopathology.

\section{Conflict of Interest}

The authors declare that there are no conflicts of interest. 


\section{Reference}

1 Aytac IA, Araujo AB, Johannes CB, Kleinman KP, Mckinlay JB (2000) Socioeconomic factors and incidence of erectile dysfunction: Findings of the longitudinal massachussetts male aging study. Soc Sci Med 51: 771-778.

2 National Institutes of Health, Impotence (1992) NIH Consensus Statement 10: 1-31.

3 Montague DK, Jarow JP, Broderick GA, Dmochowski RR, Heaton JP, et al. (2005) The management of erectile dysfunction: an AUA update. J Urol 174: 230-239.

4 Gresser U, Gleiter CH (2002)Erectile dysfunction: comparison of efficacy and side effects of the PDE- 5 inhibitors sildenafil, vardenafil and tadalafil-review of the literature. Eur J Med Res 7: 435-446.

5 Marmor MF, Kessler R (1999) Sildenafil (Viagra) and ophthalmology. Surv Ophthalmol 44: 153-162.

6 Burnett AL (1997) Nitirc oxide in the penis: physiology and pathology. J Urol 157: 320-324.

7 Goldstein I, Lue TF, Padma-Nathan H, Rosen RC, Steers WD, et al. (1998) Oral sildenafil in the treatment of erectile dysfunction. New Engl J Med 338: 1397-1404.

8 Morales A, Heaton JP, Johnston B, Adams M (1995) Oral and topical treatment of erectile dysfunction: present and future. Urol Clin North Am 22: 879-886.

9 Williams SK, Melman A (2012) Novel therapeutic targets for erectile dysfunction. Maturitas 71: 20-27.

10 Carson CC, Rosenberg M, Kissel J, Wong DG (2014) Tadalafil-A therapeutic option in the management of BPH-LUTS. Int J Clin Pract 68: 94-103.

11 Preston IR, Hinzmann B, Heinz S, Gall H, Jenkins D, et al. (2016) An international physician survey of pulmonary arterial hypertension management. Pulm Circ 6: 338-346.

12 Teich AF, Sakurai M, Patel M, Holman C, Saeed F, Fiorito J, Arancio $\mathrm{O}$ (2016) PDE5 Exists in Human Neurons and is a Viable Therapeutic Target for Neurologic Disease. J Alzheimers Dis. 52(1):295-302.
13 Hatzimouratidis K, Hatzichristou DG (2008) Looking to the future for erectile dysfunction therapies. Drugs. 68: 231-250.

14 Chen X, Wang N, Liu Y, Zhang T, Zhu L, et al. (2014) Yonkenafil: a novel phosphodiesterase type 5 inhibitor induces neuronal network potentiation by a cGMP-dependent Nogo-R axis in acute experimental stroke. Exp Neurol 261: 267-277.

15 Zhu L, Yang JY, Xue X, Dong YX, Liu Y, et al. ( 2015) A novel phosphodiesterase- 5 inhibitor: yonkenafil modulates neurogenesis, gliosis to improve cognitive function and ameliorates amyloid burden in an APP/PS1 transgenic mice model. Mech Ageing Dev 150: 34-45.

16 Zhao S, Yang J, Wang L, Peng S, Yin J, et al. (2015) NF-kappaB upregulates type 5 phosphodiesterase in $\mathrm{N} 9$ microglial cells: inhibition by sildenafil and yonkenafil. Mol Neurobiol 53: 2647-2658.

17 Mas M, Garcia-Giralda L, Rey JR, Martinez Salamanca JI, Guirao L, et al. (2011) Evaluating a continuous medical education program to improve general practitioners awareness and practice on erectile dysfunction as a cardiovascular risk factor. J Sex Med 8: 1585-1593.

18 Wang J, Jiang $Y$, Wang $Y$, Tang $Y$, Teng G, et al. ( 2008) A rapid and sensitive LC-MS/MS assay to quantify yonkenafil in rat plasma with application to preclinical pharmacokinetics studies. J Pharm Biomed Anal 47: 985-989.

19 McCullough AR, Barada JH, Fawzy A, Guay AT, Hatzichristou D ( 2002) Achieving treatment optimization with sildenafil citrate (Viagra) in patients with erectile dysfunction. Urology 60: 28-38.

20 Abbott D, Comby P, Charuel C, Graepel P, Hanton G, et al. ( 2004) Preclinical saftey profile of sildenafil. Int J Impt Res 16: 498-504.

21 Gao Y, Li C, Kang L, Hang B, Yan M, et al. ( 2014) A subchronic toxicity study, preceded by an in utero exposure phase, with refined arachidonic acid-rich oil (RAO) derived from Mortierella alpine XM027 in rats. Regul Toxicol Pharmacol 70: 696-703.

22 Bischoff B (2004) Vardenafil preclinical trial data: potency, pharmacodynamics, pharmacokinetics, and adverse events. Int J Impt Res 16: S34-S37.

23 Limin Ma, Johnsen N, Hellstrom WJ (2010) Avanafil, a new rapidonset phosphodiesterase 5 inhibitor for the treatment of erectile dysfunction. Expert Opin Investig Drugs 19:1427-1437. 